\title{
PREDICTORS OF ANTIPSYCHOTIC \\ POLYPHARMACY IN AN ACUTE-CARE TEACHING HOSPITAL
}

\author{
Deanna Bice, PharmD, BCPS \\ Clinical Pharmacy Specialist, Critical Care \\ Vidant Medical Center \\ Greenville, North Carolina \\ d-bice@onu.edu \\ Chris Paxos, PharmD, BCPP, BCPS, CGP \\ Pharmacotherapy Specialist, Psychiatric Medicine \\ Akron General Medical Center \\ Akron, Ohio \\ cpaxos@neomed.edu \\ Jessica L. Boss, PharmD, BCPS, CGP \\ Pharmacotherapy Specialist, Palliative Care \\ Akron General Medical Center \\ Akron, Ohio \\ jboss1@neomed.edu \\ John M. Moorman, PharmD, BCPS \\ Pharmacotherapy Specialist, Endocrinology \\ Akron General Medical Center \\ Akron, Ohio \\ jmoorman@neomed.edu \\ Dustin K. Blakeslee, DO, FAPA \\ Medical Director, Consultation/Liaison Psychiatry \\ Akron General Medical Center \\ Akron, Ohio dblakeslee@ akrongeneral.org
}

Corresponding Author:

\author{
Chris Paxos, PharmD, BCPP, BCPS, CGP \\ Pharmacotherapy Specialist, Psychiatric Medicine \\ Akron General Medical Center \\ 1 Akron General Avenue \\ Akron, OH 44307 \\ Email: cpaxos@neomed.edu \\ Phone: 330-325-6443 \\ Fax: 330-325-5951
}

Introduction-Antipsychotic polypharmacy increases the risk of adverse reactions, interactions, and cost. Data for the use of antipsychotic polypharmacy are limited. The Joint Commission's Hospital Based Inpatient Psychiatric Services (HBIPS) recently established a set of quality measures evaluating inpatient psychiatric care, including the rate of antipsychotic polypharmacy at discharge. 
Methods - A retrospective medical record review was approved by the Institutional Review Board. Admission records were used to identify patients 18 years or older who were discharged from an inpatient psychiatric unit from January 1, 2012 to June 30, 2012. Exclusion criteria included patients not discharged on antipsychotics, pregnancy, length of stay not more than three days, elopement or failing to return from leave, or those who expired. Patients were divided into two groups: those discharged on antipsychotic monotherapy or antipsychotic polypharmacy. The objective of this study was to determine the association between patient-specific factors and the presence of antipsychotic polypharmacy at discharge. A secondary objective was determining if appropriate justification for antipsychotic polypharmacy at discharge was documented.

Results-Of 901 inpatient psychiatric admissions, 442 patients were included. There were 362 patients discharged on antipsychotic monotherapy and 80 patients discharged on antipsychotic polypharmacy. Five characteristics were significantly associated with antipsychotic polypharmacy at discharge: Medicare insurance (OR 2.156, 95\% CI 1.279-3.636), psychotic disorders (OR 3.665, 95\% CI 1.999-6.789), greater than one antipsychotic on admission (OR 27.179, 95\% CI 13.895-53.668), quetiapine at discharge (OR 2.3, 95\% CI 1.355-3.901), and paliperidone at discharge (OR 2.415, 95\% CI 1.38-4.219). Of the 80 patients discharged on antipsychotic polypharmacy, $83.7 \%$ did not have appropriate justification for the polypharmacy documented.

Conclusions - The patient-specific factors of Medicare, psychotic disorders, greater than one antipsychotic on admission, and quetiapine and paliperidone were significantly associated with antipsychotic polypharmacy at discharge. For patients discharged on multiple antipsychotics, supporting documentation was infrequent.

Keywords_psychiatry; antipsychotic; polypharmacy 


\section{Introduction}

Antipsychotic polypharmacy, the concurrent use of two or more antipsychotic medications, is common in clinical practice. Rates of polypharmacy for the treatment of psychiatric disorders have been estimated to be as high in $50 \%$ in some settings (Barnes \& Paton, 2011). A review of medical records completed at an academic medical center found approximately $10 \%$ of patients had been prescribed two or more antipsychotics at discharge (Huffman et al, 2011). A study conducted at Veterans Affairs facilities revealed $20 \%$ and $9.5 \%$ of patients received antipsychotic polypharmacy for greater or equal to 30 days or 90 days, respectively (Kreyenbuhl et al, 2006).

Despite this common practice, minimal data support the routine use of antipsychotic polypharmacy. The available literature is predominately limited to augmentation of clozapine therapy in treatment-resistant patients; however, small sample sizes, variability in defining treatment-resistance, and differing study outcomes limit the utility of these findings (Porcelli, Balzarro, \& Serretti, 2012). Furthermore, concurrent use of two or more antipsychotic agents may increase overall drug cost, adverse effects, and drug interactions (Lochmann van Bennekom, Gijsman, \& Zitman, 2013). A case-control trial of psychiatric inpatients evaluated length of stay, change in clinical improvement scores, and adverse effects in those receiving either antipsychotic monotherapy or polypharmacy (Centorrino et al, 2004). Length of stay was $55 \%$ longer in the polypharmacy groups; however, there was no statistical difference in clinical improvement scores (Centorrino et al, 2004). Although not statistically significant, adverse effects were more common in the polypharmacy group (Centorrino et al, 2004). For example, patients experiencing at least one extrapyramidal side effect in the polypharmacy and monotherapy groups were $30.0 \%$ and $24.3 \%$, respectively (Centorrino et al, 2004).

The increased risk of adverse effects and modest data supporting use are reflected in major practice guidelines from the American Psychiatric Association (APA), Texas Implementation of Medication Algorithms (TIMA), and the Patient Outcomes Research Team (PORT) recommendations (Buchanan et al, 2010; Moore et al, 2007; APA, 2004). All practice guidelines consistently advocate for the use of monotherapy for the treatment of schizophrenia and for the use of clozapine for treatment-resistant disease (Buchanan et al, 2010; Moore et al, 2007; APA, 2004). The APA practice guidelines suggest adequate documentation that a patient has not sufficiently benefited from monotherapy with either component of combination therapy; moreover, the guidelines discuss the negative impact of polypharmacy regimens on adverse effects, drug interactions, cost, and medication adherence (APA, 2004).

To address the issue of antipsychotic polypharmacy in clinical practice, the Joint Commission and the National Association of State Mental Health Program Directors established measures to evaluate inpatient psychiatric care called the Hospital Based Inpatient Psychiatric Services (HBIPS) (The Joint Commission, 2012). Two of these quality measures, HBIPS-4 and HBIPS-5, specifically address the use of antipsychotic polypharmacy (The Joint Commission, 2012). HBIPS-4 assesses the rate of multiple antipsychotics prescribed at discharge, while HBIPS-5 assesses whether appropriate justification was provided for 
those patients discharged on multiple antipsychotics (The Joint Commission, 2012). A previous study evaluated patientspecific factors associated with antipsychotic polypharmacy at discharge in an academic medical center (Huffman, 2011). This study was conducted to determine whether patient-specific factors can be identified in a community not-forprofit hospital population.

\section{Methods}

A retrospective medical record review was conducted at Akron General Medical Center (AGMC), a 532 bed not-for-profit community teaching hospital with 55 licensed inpatient psychiatric beds. Patients at least 18 years of age and admitted to an inpatient psychiatric unit at AGMC between January 2012 and June 2012 were included. This represents the time period immediately prior to staff education regarding the HBIPS quality measures. Exclusion criteria mirrored those in the HBIPS-5 measure and included the following: patients who were pregnant, discharged on zero antipsychotic medications, had a length of stay less than or equal to three days, or were discharged due to elopement or failing to return from leave. The study was approved by the AGMC Institutional Review Board.

The primary objective was to determine whether an association exists between patient-specific factors and the presence of antipsychotic polypharmacy at hospital discharge. The patient-specific factors analyzed included gender, race, third-party insurance, psychiatric diagnoses, antipsychotics prescribed upon admission and discharge, number of psychiatric admissions during the study period, and discharge location. Secondary outcomes included determining the percentage of patients discharged on antipsychotic polypharmacy and describing whether appropriate justification was documented. Appropriate justification, as defined by HBIPS-5, included patients with a documented history of at least three failed trials of antipsychotic monotherapy, patients with a planned taper to monotherapy as an outpatient or cross taper in progress at time of discharge, and the augmentation of clozapine with antipsychotic therapy (The Joint Commission, 2012).

All data were collected via electronic patient medical records and recorded within a Microsoft Access ${ }^{\circledR}$ database. Patients received a numerical identifier to maintain confidentiality. Demographic data and outcome measures were analyzed using Fisher's exact tests for categorical data and Student's t-tests for continuous data. Odds ratios (OR) with $95 \%$ confidence intervals (CI) were calculated using Fisher's exact tests for predictor variable analyses. The secondary outcome was analyzed using descriptive statistics.

\section{Results}

A total of 901 patients were admitted to an inpatient psychiatric unit at AGMC during the study timeframe. Of these admissions, 459 were excluded from the study for the reasons of length of stay less than or equal to three days (270 patients) and zero antipsychotics prescribed at discharge (189 patients). Of the 442 patients included in the study, 362 patients were discharged on antipsychotic monotherapy, and 80 patients were discharged on antipsychotic polypharmacy as detailed in Figure 1. 
Figure 1. Group assignment

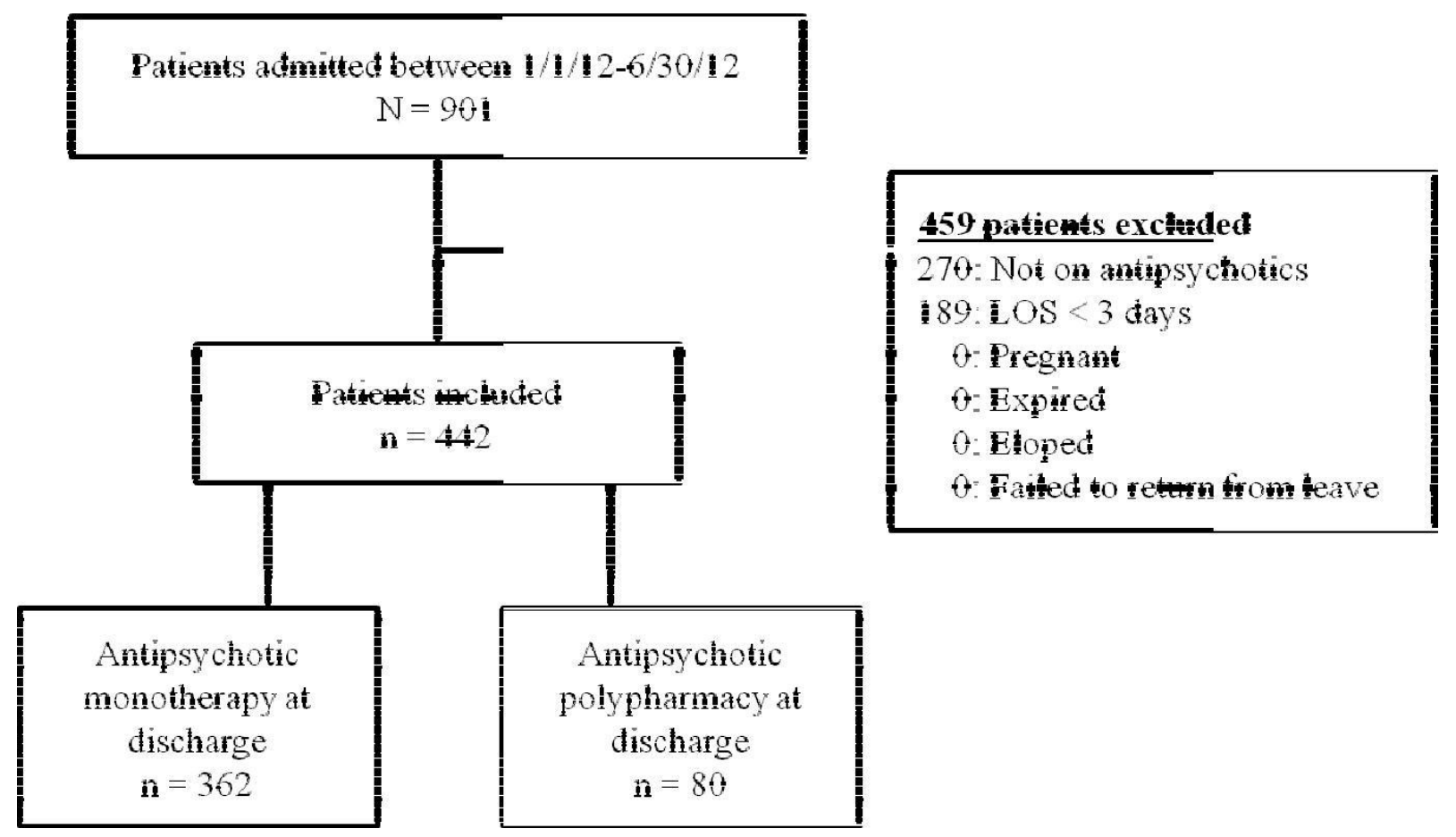

Patient demographics were analyzed to determine if differences existed between groups (Table 1). Patients in the antipsy chotic polypharmacy group were significantly older, more often had Medicare, had longer lengthss of stay, and had a higher number of antipsycho tics prescribed on admission than the antipsycho tic monotherapy group. Conversely, more patients in the monotherapy group had private insurance. 
Table 1. Patient demographics

\begin{tabular}{|l|c|c|c|}
\hline & $\begin{array}{c}\text { Monotherapy } \\
(\mathrm{n}=362)\end{array}$ & $\begin{array}{c}\text { Polypharmacy } \\
(\mathrm{n}=80)\end{array}$ & p-value \\
\hline Male (n, \%) & $145(40 \%)$ & $38(47.5 \%)$ & 0.259 \\
\hline Age (mean \pm S.D.) & $42.9 \pm 15.1$ & $47.6 \pm 15.8$ & 0.013 \\
\hline Race (n, \%) & \multicolumn{3}{|l|}{} \\
\hline White & $276(76.2 \%)$ & $59(73.8 \%)$ & 0.666 \\
\hline Black & $75(20.7)$ & $21(26.3)$ & 0.295 \\
\hline Asian & $1(0.3)$ & $0(0)$ & 1.0 \\
\hline Other & $10(2.8)$ & $0(0)$ & 0.22 \\
\hline Insurance (n, \%) & \multicolumn{3}{|l|}{} \\
\hline \multicolumn{1}{|l|}{ Medicaid } & $153(42.3 \%)$ & $28(35 \%)$ & 0.259 \\
\hline \multicolumn{1}{|l|}{ Medicare } & $107(29.6)$ & $38(47.5)$ & 0.002 \\
\hline \multicolumn{1}{|l|}{ Private } & $93(25.7)$ & $9(11.3)$ & 0.005 \\
\hline None & $9(2.5)$ & $5(6.3)$ & 0.147 \\
\hline $\begin{array}{l}\text { Length of stay } \\
\text { (mean } \pm \text { S.D.) }\end{array}$ & $8.5 \pm 5.8$ & $12.4 \pm 8.5$ & $<0.0001$ \\
\hline $\begin{array}{l}\text { Number of antipsychotics } \\
\text { on admission } \\
\text { (mean } \pm \text { S.D.) }\end{array}$ & $1.1 \pm 0.4$ & $1.7 \pm 0.6$ & $<0.0001$ \\
\hline $\begin{array}{l}\text { Number of psychiatric } \\
\text { admissions } \\
\text { (mean } \pm \text { S.D.) }\end{array}$ & $1.4 \pm 0.8$ & $1.5 \pm 0.7$ & 0.302 \\
\hline P-values were considered significant if $<0.05 ;$ S.D. $=$ standard deviation & \\
\hline
\end{tabular}

Five of the pre-identified patient-specific factors were significantly associated with antipsychotic polypharmacy at discharge (Figure 2). Patients with Medicare (OR 2.156, 95\% CI 1.279-3.636), a psychotic disorder (OR 3.665, 95\% CI 1.999-6.789), greater than one antipsychotic prescribed on admission (OR 27.179, 95\% CI 13.89553.668), quetiapine prescribed at discharge (OR 2.3, 95\% CI 1.355-3.901), or paliperidone prescribed at discharge (OR $2.415, \quad 95 \%$ CI 1.38-4.219) were significantly associated with the presence of antipsychotic polypharmacy at discharge. Two patient-specific factors were significantly associated with the presence of antipsychotic monotherapy at discharge: private insurance (OR 0.367, 95\% CI 0.164-0.795) and bipolar disorder (OR $0.251,95 \%$ CI $0.108-0.562)$. There was no association with either group for any of the other patient-specific characteristics. 
Figure 2. Predictors of antipsychotic polypharmacy on hospital discharge

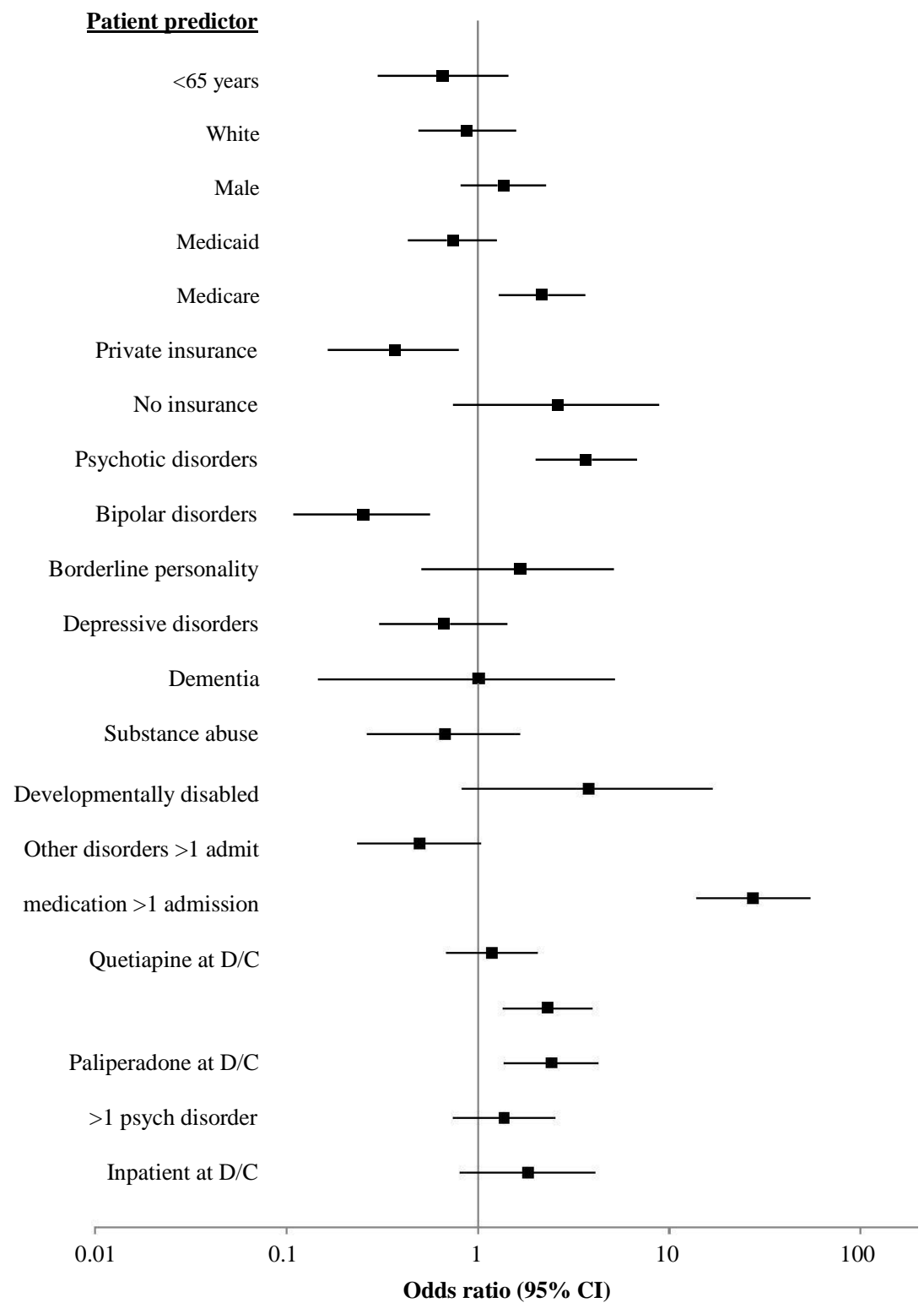

Of the 442 patients included in the study, 80 patients (18\%) were discharged from the hospital receiving antipsychotic polypharmacy. Of the patients discharged on antipsychotic polypharmacy, the majority $(83.7 \%)$ did not have appropriate justification as defined by HBIPS-5 documented in the medical chart. Of the thirteen patients that had an appropriate justification documented, six (7.5\%) were receiving clozapine therapy which was being augmented with a second antipsychotic, five $(6.3 \%)$ had a planned taper to monotherapy as an outpatient or were undergoing a cross taper at time of discharge, and two (2.5\%) had at least three failed trials of antipsychotic monotherapy documented. 


\section{Discussion}

This study demonstrated an association between five patient-specific factors and the presence of antipsychotic polypharmacy at discharge. Medicare as a significant factor for antipsychotic polypharmacy at discharge was unexpected given no association between polypharmacy and age; however, the association may represent patients with Medicare disability benefits secondary to their psychiatric disorder. Furthermore, these patients may be more connected to the community mental health system and have more severe and persistent mental illness. Psychotic disorders were a patient-specific factor for antipsychotic polypharmacy at discharge. This finding may be explained given the fact that antipsychotics are the cornerstone of pharmacotherapy for the treatment of psychosis. A patient being prescribed at least two antipsychotics on admission was also significantly associated with polypharmacy, and this likely occurred secondary to short inpatient hospitalizations which may not allow for time to change a regimen to monotherapy by discharge. Furthermore, admission with multiple antipsychotics may also represent patients with more severe symptomatology. Finally, the presence of either quetiapine or paliperidone - both second generation antipsychotics - was associated with antipsychotic polypharmacy at discharge. Quetiapine association with antipsychotic polypharmacy at discharge may be explained by its numerous U.S. Food and Drug Administration approved indications, including schizophrenia, manic or depressive episodes associated with bipolar disorder, or major depressive disorder as adjunctive therapy to antidepressants (Seroquel $^{\circledR}, 2013$; Seroquel XR $\left.{ }^{\circledR}, 2013\right)$. Additionally, quetiapine is utilized extensively for off-label indications (Philip et al, 2008). Finally, paliperidone and its association with antipsychotic polypharmacy at discharge are likely explained by the high usage of the paliperidone long-acting, intramuscular formulation at AGMC.

Two characteristics were associated with antipsychotic monotherapy at discharge: private insurance and bipolar disorder. Private insurance may potentially reflect a higher degree of functioning and less severe symptomatology, leading to the association with antipsychotic monotherapy, although other explanations are quite possible. Bipolar disorder may be associated with antipsychotic monotherapy as symptom improvement is often achieved with traditional mood stabilizers. These are often utilized alone or in combination with a second mood stabilizer or an antipsychotic, making antipsychotic polypharmacy potentially less likely.

There was a high incidence of antipsychotic polypharmacy at our institution, which is consistent with rates cited in previous studies (Gallego et al, 2012; Ganguly et al, 2004). In addition, there was limited documentation of appropriate justification for patients that were prescribed antipsychotic polypharmacy at discharge. While this rate of documentation is low, there is currently no minimum percentage required by the Joint Commission's HBIPS quality measures. This study has established a baseline rate of documentation at our institution, and enhanced clinician education is underway in order to improve documentation rates.

There were several limitations to this study. A major limitation includes the retrospective design; patient adherence to antipsychotic therapy could not be assessed, and we were forced to rely on accuracy of documentation within the medical record (i.e., medication reconciliation, polypharmacy justification). Although documentation regarding 
antipsychotic polypharmacy justification was lacking in our study, the possibility exists that patients may have had appropriate rationale for the prescribed polypharmacy at discharge in the absence of documentation. Lastly, patients may have been admitted to other area psychiatric institutions within the study period which could have affected the patient-specific factor related to the number of psychiatric admissions.

\section{Conclusions}

Patient-specific factors significantly associated with the presence of antipsychotic polypharmacy at discharge included Medicare insurance, the presence of a psychotic disorder, more than one antipsychotic upon admission, and either quetiapine or paliperidone prescribed at discharge. Patient-specific factors may assist clinicians in identifying patients at risk for antipsychotic polypharmacy at discharge, a quality measure tracked by HBIPS, in order to evaluate inpatient psychiatric care. Clinician education regarding this quality measure, as well as the HBIPS measure evaluating the appropriate justification of antipsychotic polypharmacy, is currently underway. This study enhances the limited literature available regarding predictors of antipsychotic polypharmacy at discharge in the community hospital setting.

\section{Acknowledgement}

The authors would like to thank Dr. Jeffrey L. Moore, without whom this research would not have been possible. 


\section{References}

American Psychiatric Association. (2004) Practice guidelines for the treatment of patients with schizophrenia. Second edition. Am J Psychiatry, 1-114.

Barnes, T.R., and Paton, C. (2011) Antipsychotic polypharmacy in schizophrenia: benefits and risks. CNS Drugs, 25:383-399.

Buchanan, R.W., Kreyenbuhl, J., Kelly, D.L., Noel, J.M., Boggs, D.L., Fischer, B.A., et al. (2010) The 2009 schizophrenia PORT psychopharmacological treatment recommendations and summary statements. Schizophr Bull, 36:71-93.

Centorrino, F., Gören, J.L., Hennen, J., Salvatore, P., Kelleher, J.P., Baldessarini, R. J. (2004) Multiple versus single antipsychotic agents for hospitalized psychiatric patients: case-control study of risks versus benefits. Am J Psychiatry, 161:700-706.

Gallego, J.A., Bonetti, J., Zhang, J., Kane, J.M., \& Correll, C.U. (2012) Prevalence and correlates of antipsychotic polypharmacy: a systematic review and meta-regression of global and regional trends from the 1970s to 2009. Schizophr Res, 138:18-28.

Ganguly, R., Kotzan, J.A., Miller, L.S., Kennedy, K., \& Martin, B.C. (2004) Prevalence, trends, and factors associated with antipsychotic polypharmacy among Medicaid-eligible schizophrenia patients, 1998-2000. J Clin Psychiatry, 65:13771388.

Huffman, J.C., Chang, T.E., Durham, L.E., and Weiss, A.P. (2011) Antipsychotic polytherapy on an inpatient psychiatric unit: how does clinical practice coincide with Joint Commission guidelines? Gen Hosp Psych, 33:501-508.
Kreyenbuhl, J., Valenstein, M., McCarthy, J.F., Ganoczy, D., and Blow, F.C. (2006) Long-term combination antipsychotic treatment in VA patients with schizophrenia. Schiz Res, 84;90-99.

Lochmann van Bennekom, M.W., Gijsman, H.J., Zitman, F.G. (2013) Antipsychotic polypharmacy in psychotic disorders: a critical review of neurobiology, efficacy, tolerability and cost effectiveness. J Psychopharmacol, 27:327-336.

Moore, T.A., Buchanan, R.W., Buckley, P.F., Chiles, J.A., Conley, R.R., Crismon, L.M., et al. (2007) The Texas Medication Algorithm Project antipsychotic algorithm for schizophrenia: 2006 update. J Clin Psychiatry, 68:1751-62.

Philip, N.S., Mello, K., Carpenter, L.L., Tyrka, A.R., Price, L.H. (2008) Patterns of quetiapine use in psychiatric inpatients: an examination of off-label use. Ann Clin Psychiatry, 20:15-20.

Porcelli, S., Balzarro, B., Serretti, A. (2012). Clozapine resistance: augmentation strategies. Eur Neuropsychopharm, 22:165-182.

Seroquel $^{\circledR}$ [package insert]. Wilmington, DE: AstraZeneca Pharmace uticals LP; October 2013. Seroquel XR ${ }^{\circledR}$ [package insert]. Wilmington, DE: AstraZeneca Pharmaceuticals LP; October 2013.

The Joint Commission. Joint Commission national quality core measures - hospital based inpatient psychiatric services (HBIPS) core measure set; 2012. Available at http://manual.jointcommission.org/r eleases/TJC2012B (Accessed February 16, 2015). 